\title{
Damping of standing slow waves in hot coronal loops
}

\section{Leonardo Di G. Sigalotti ${ }^{1}$, César A. Mendoza-Briceño ${ }^{2}$ and Marialejandra Luna-Cardozo ${ }^{3}$}

\author{
${ }^{1}$ Instituto Venezolano de Investigaciones Científicas (IVIC), Centro de Física, Laboratorio de \\ Física de Fluidos y Plasmas, Caracas-Venezuela \\ email: leonardo.sigalotti@gmail.com \\ ${ }^{2}$ Centro de Física Fundamental, Facultad de Ciencias, \\ Universidad de los Andes, Merida-Venezuela \\ email: cesar@ula.ve
}

${ }^{3} \mathrm{SP}^{2} \mathrm{RC}$, Department of Applied Mathematics, University of Sheffield, Sheffield S3 7RH, UK email: m.luna@sheffield.ac.uk

\begin{abstract}
The damping of standing slow mode oscillations in hot $(T>6 \mathrm{MK})$ coronal loops is described in the linear limit. The effects of energy dissipation by thermal conduction, viscosity, and radiative losses and gains are examined for both stratified and nonstratified loops. We find that thermal conduction acts on the way of increasing the period of the oscillations over the sound crossing time, whereas the decay times are mostly determined by viscous dissipation. Thermal conduction alone results in slower damping of the density and velocity waves compared to the observations. Only when viscosity is added do these waves damp out at the same rate of the observed SUMER loop oscillations. In the linear limit, the periods and decay times are barely affected by gravity.
\end{abstract}

Keywords. Sun: corona, Sun: oscillations, (magnetohydrodynamics:) MHD, waves

\section{Introduction}

Coronal loop oscillations may occur as a result of both standing and propagating waves. In particular, far-ultraviolet observations with the aid of the SUMER spectrometer have revealed strongly damped, large Doppler shift oscillations in hot $(T>6 \mathrm{MK})$ coronal loops (Kliem et al. 2002; Wang et al. 2002a, 2002b, 2003a, Banerjee et al. 2007). Such oscillations were interpreted in terms of standing slow waves (Ofman \& Wang 2002; Wang et al. 2003b, 2005) and have periods in the range of 8.6 to 32.3 minutes and decay times between 3.1 and 42.3 minutes (Wang et al. 2005). Most recent studies of hot loop oscillations have relied either on linear perturbation analyses or numerical simulations of the one-dimensional (1D) acoustic wave. In particular, Ofman \& Wang (2002) simulated numerically the damping of standing slow waves for typical solar coronal conditions. They found that thermal conduction is the dominant dissipation mechanism based on the realization that only the conductive timescale is of the same order of the observed decay times.

The influence of gravitational stratification on the decay of standing slow modes was considered by Mendoza-Briceño, Erdélyi, \& Sigalotti (2004). They found that the wave periods remain almost unaffected by gravity, while the damping times are reduced by $\sim 10 \%-20 \%$ compared to the nonstratified loops because of enhanced viscous dissipation induced by gravity. Based on linearized solutions to the Klein-Gordon wave equation, Roberts (2006) showed that stratification introduces an effective loop length which is by far larger than the observed lengths of SUMER hot loops, implying that in the linear 
limit the effects of stratification on the period of slow modes are small. Whereas all the above results apply to isothermal loops, Taroyan et al. (2005) considered the additional effect of temperature inhomogeneities along the loop on standing slow wave dissipation, including the thermal conduction and optically thin radiative losses as the only sinks of energy. Recently, Taroyan et al. (2007) investigated longitudinal loop oscillations with predicted observables, like EUV line profiles, as response to a microflare.

In this paper, we reconsider the dissipation of standing slow MHD waves in isothermal, hot $(T>6 \mathrm{MK})$ coronal loop models, including the effects of gravitational stratification, thermal conduction, viscosity, heating, and optically thin radiation losses. The implications of each of these mechanisms on wave damping are examined by solving the linearized 1D coupled system of loop equations, using an approach similar in spirit to that presented by De Moortel \& Hood $(2003,2004)$.

\section{Loop Models}

Since the plasma dynamics in a coronal loop is dominated by the geometry of the magnetic field, we assume that both plasma motion and heat conduction (due to electron diffusion) inside the loop are essentially guided along the magnetic field lines. Hence, to a good approximation, the loop can be represented by a 1D semicircular magnetic flux tube of constant cross-sectional area with footpoints fixed in the chromosphere. With these assumptions, the governing loop equations have the form of the $1 \mathrm{D}$ hydrodynamic conservation laws. We consider the effects of solar gravity, heat conduction, viscosity, and optically thin radiation. We consider two sets of loop model calculations. One set deals with perfectly homogeneous loops, in which the initial density and pressure are constant, while the other set corresponds to stratified loop models. In both cases, the temperature is taken to be uniform along the loop.

For the present models we vary three parameters, namely the initial loop temperature $\left(6.3 \leqslant T_{0} \leqslant 10 \mathrm{MK}\right)$, the loop length $(50 \leqslant L \leqslant 400 \mathrm{Mm})$, and the initial velocity amplitude $\left(20 \leqslant v_{0} \leqslant 200 \mathrm{~km} \mathrm{~s}^{-1}\right)$.

Following De Moortel \& Hood (2003, 2004), the effects of compressive viscosity, heat conduction, and radiative losses/gains can be quantified in terms of the dimensionless parameters, namely the Reynolds number $R, \varepsilon=\eta /\left(\rho L c_{\mathrm{s}}\right)=1 / R$, the thermal ratio $d=1 / \gamma\left[\tau_{\mathrm{s}} / \tau_{\text {cond }}\right]$, which is the ratio of the sound travel time and the thermal conduction timescale, and the radiation ratio $r=\tau_{\mathrm{s}} / \tau_{\mathrm{rad}}$, which is the ratio of the sound travel time and the radiation timescale.

The values of the above parameters and timescales for varying loop lengths and temperatures are listed in Table 1 for the homogeneous loops. The same values also hold for the stratified models, where the reference density $\left(\rho_{0}\right)$ and pressure $\left(p_{0}\right)$ now refer to the footpoint values. Note that $\varepsilon$ and $d$ increase with the loop temperature and decrease with the loop length, while the converse occurs for the radiation ratio $r$. We see that $\tau_{\mathrm{s}}$ and $\tau_{\text {cond }}$ are of the same order and become fairly comparable in long loops. Moreover, $\tau_{\text {visc }} \approx 37.54 \tau_{\text {cond }}$ in all cases, implying that for typical conditions of the hot solar corona the conductive timescale is much shorter than the viscous time. The radiation timescales are not shown in Table 1 because they are by far much longer than the other times, taking values from $\sim 1.8 \times 10^{4}$ minutes (for $T=6.3 \mathrm{MK}$ ) to about $7.4 \times 10^{4}$ minutes (for $T=10 \mathrm{MK}$ ). Also, the conductive times are well within the range of observed decay times of standing slow waves, suggesting that wave dissipation takes place over the thermal conduction timescale. 
Table 1. Parameters and characteristic times as functions of the loop length $(L)$ and temperature $(T)$. The dimensionless numbers $\varepsilon, d$, and $r$ quantify the importance of viscosity, thermal conduction, and radiation respectively, while $\tau_{\mathrm{s}}$ is the sound crossing time, $\tau_{\mathrm{visc}}$ is the viscous time, and $\tau_{\text {cond }}$ is the heat-conduction time

\begin{tabular}{|c|c|c|c|c|c|c|}
\hline $\begin{array}{c}L \\
(\mathrm{Mm})\end{array}$ & $\varepsilon$ & $d$ & $r$ & $\begin{array}{c}\tau_{\mathrm{s}} \\
(\min )\end{array}$ & $\begin{array}{l}\tau_{\mathrm{visc}} \\
(\mathrm{min})\end{array}$ & $\begin{array}{l}\tau_{\text {cond }} \\
(\mathrm{min})\end{array}$ \\
\hline \multicolumn{7}{|c|}{$T=6.3 \mathrm{MK}$} \\
\hline 50 & $7.97(-2)$ & 2.39 & $1.18(-4)$ & 2.1847 & 20.56 & 0.55 \\
\hline 100 & $3.98(-2)$ & 1.20 & $2.37(-4)$ & 4.3693 & 82.24 & 2.19 \\
\hline 200 & $1.99(-2)$ & 0.60 & $4.73(-4)$ & 8.7386 & 328.96 & 8.76 \\
\hline 300 & $1.33(-2)$ & 0.40 & $7.10(-4)$ & 13.1080 & 740.17 & 19.72 \\
\hline 400 & $9.96(-3)$ & 0.30 & $\begin{array}{c}9.46(-4) \\
T=8 \mathrm{MK}\end{array}$ & 17.4773 & 1315.86 & 35.05 \\
\hline 50 & 0.16 & 4.90 & $5.13(-5)$ & 1.9387 & 8.91 & 0.24 \\
\hline 100 & $8.16(-2)$ & 2.45 & $1.03(-4)$ & 3.8774 & 35.64 & 0.95 \\
\hline 200 & $4.08(-2)$ & 1.23 & $2.05(-4)$ & 7.7548 & 142.57 & 3.80 \\
\hline 300 & $2.72(-2)$ & 0.82 & $3.08(-4)$ & 11.6322 & 320.78 & 8.55 \\
\hline 400 & $2.04(-2)$ & 0.61 & $\begin{array}{c}4.10(-4) \\
T=10 \mathrm{MK}\end{array}$ & 15.5096 & 570.28 & 15.19 \\
\hline 50 & 0.32 & 9.57 & $2.35(-5)$ & & 4.08 & 0.11 \\
\hline 100 & 0.16 & 4.79 & $4.70(-5)$ & 3.4680 & 16.32 & 0.43 \\
\hline 200 & $7.97(-2)$ & 2.39 & $9.39(-5)$ & 6.9361 & 65.29 & 1.74 \\
\hline 300 & $5.31(-2)$ & 1.60 & $1.41(-4)$ & 10.4041 & 146.90 & 3.91 \\
\hline 400 & $3.98(-2)$ & 1.20 & $1.88(-4)$ & 13.8722 & 261.16 & 6.96 \\
\hline
\end{tabular}

\section{Linear Perturbation Analysis}

The governing equations are linearized under the assumption that the unperturbed state consists of a stationary $\left(v_{0}=0\right)$ plasma in equilibrium at uniform temperature $T_{0}$. For a homogeneous loop, the unperturbed state is independent of position, while in the stratified case the stationary loop is in hydrostatic equilibrium. The resulting set of linear equations are solved by expressing the spatial dependence of the perturbed variables in terms of a Fourier series expansion, where the expansion coefficients depend only on time and denote the Fourier mode amplitudes. Decoupling of the spatial dependence from time allows exact evaluation of the spatial derivatives and transforms the linearized equations into a set of $6 n$ ordinary differential equations for the time rate of change of the $6 n$ perturbation amplitudes, where $n$ is an integer mode number. The time integration is performed using a predictor-corrector solver so that the evolution is temporally secondorder accurate.

\subsection{Linear Wave Dissipation}

We first consider the effects of wave dissipation on homogeneous loops and then extend the analysis to the case of stratified loops.

\subsubsection{Homogeneous Loops}

Since the effects of radiation losses and gains on wave dissipation are negligible, we shall focus our discussion in terms of the dimensionless ratios $\varepsilon$ and $d$ only.

Viscosity is found to result in a reduction of the wave frequency and hence in an increase of the period. In order to see this in quantitative terms, let us consider the 

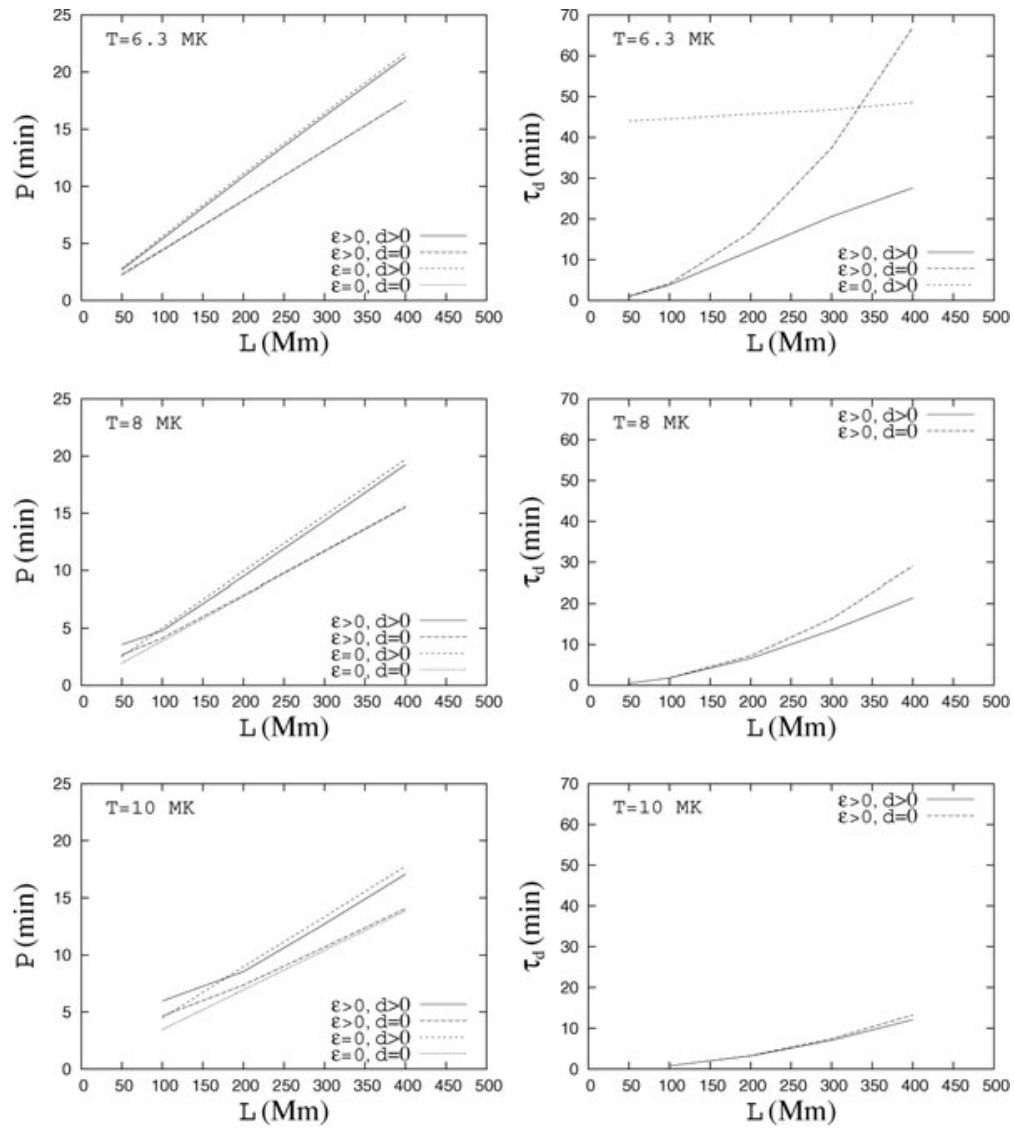

Figure 1. Wave period ( $P$ : left panels) and decay time $\left(\tau_{\mathrm{d}}\right.$ : right panels) as functions of length $L$ in oscillating homogeneous loops for varying temperatures and choices of the parameters $\varepsilon$ and $d$. In the middle and lower-right panels, the short-dashed curve (for $\varepsilon=0$ and $d>0$ ) falls outside the boxes and so it is not shown.

velocity wave equation when only compressive viscosity is present

$$
\frac{\partial^{2} v^{\prime}}{\partial \tilde{t}^{2}}-\frac{\partial^{2} v^{\prime}}{\partial \tilde{s}^{2}}=\frac{4}{3} \varepsilon \frac{\partial}{\partial \tilde{t}}\left(\frac{\partial^{2} v^{\prime}}{\partial \tilde{s}^{2}}\right)
$$

Setting $v^{\prime}=\tilde{v}_{0} \exp [i(\omega \tilde{t}-k \tilde{s}+\pi / 2)]$ and replacing this solution into Equation (3.1) yields the dispersion relation $\omega^{2}-i \frac{4}{3} \varepsilon k^{2} \omega-k^{2}=0$, which can be solved to give the complex frequency

$$
\omega=2 \pi\left(1-\frac{16 \pi^{2} \varepsilon^{2}}{9}\right)^{1 / 2}+i \frac{8 \pi^{2} \varepsilon}{3},
$$

where we have set $k=2 \pi$ and chosen the positive root. If $\varepsilon=0$, the imaginary part vanishes and $\omega=2 \pi$. Since $\operatorname{Im}(\omega)>0$, compressive viscosity causes the amplitude of the velocity perturbation to decrease at the rate $\exp \left(-8 \pi^{2} \varepsilon \tilde{t} / 3\right)$. The actual frequency of oscillation is given by $\operatorname{Re}(\omega)$ so that the period $[P=2 \pi / \operatorname{Re}(\omega)]$ of the second spatial harmonic is modified according to

$$
P=\tau_{\mathrm{s}}\left(1-\frac{16 \pi^{2} \varepsilon^{2}}{9}\right)^{-1 / 2} .
$$


The quantity $1-16 \pi^{2} \varepsilon^{2} / 9$ is positive for all values of $\varepsilon$ quoted in Table 1 , with the exception of $\varepsilon \approx 0.32$, which corresponds to a loop model with $T=10 \mathrm{MK}$ and $L=50$ Mm. In this case, $1-16 \pi^{2} \varepsilon^{2} / 9<0$ and the frequency becomes imaginary. This leads to an enhanced damping rate, which is consistent with the fact that for this model the oscillations undergo aperiodic critical damping. It is clear from Equation (3.3) that, in general, viscous dissipation results in an increase of the wave period. This effect is larger in short loops $(L=50 \mathrm{Mm})$, where the period increases from about $6 \%$ when $T=6.3$ MK to $\sim 37 \%$ when $T=8 \mathrm{MK}$. For comparison, in long loops $(L=400 \mathrm{Mm})$ the increase is from less than $0.1 \%(T=6.3 \mathrm{MK})$ to $\sim 1.4 \%(T=10 \mathrm{MK})$. The decay time (defined as the timescale over which the wave amplitude decreases by a factor of $e)$ is given by $\tau_{\mathrm{d}}=3 \tau_{\mathrm{s}} /\left(8 \pi^{2} \varepsilon\right)$ and takes values in the range between $\approx 0.45$ and $\approx 66.7$ minutes. Wave damping slows down at low temperatures and large lengths, suggesting that viscous dissipation is more effective in short and hot loops. We note that these damping times are much shorter than the viscous timescales (see Table 1) and compare fairly well with the observed decay times of standing slow waves.

When thermal conduction is added the wave period and the decay time also increase. The numerical solution of the set of dimesionless linearized equations for $\varepsilon=0$ and $d>0$ indicates that thermal conduction increases the period and decay time by a much larger amount than does compressive viscosity. The left panels of Figure 1 depict the dependence of the first oscillation period on loop length for different temperatures. Each plot displays four curves, corresponding to model sequences with all sinks of energy switched on $(\varepsilon>0, d>0$; solid lines), with either only compressive viscosity $(\varepsilon>0$, $d=0$; long-dashed lines) or thermal conduction $(\varepsilon=0, d>0$; short-dashed lines) allowed, and with dissipation turned off $(\varepsilon=0, d=0$; dotted lines). In general, the wave period increases with increasing loop length and decreases with increasing temperature. In particular, for $T=6.3 \mathrm{MK}$ the period rises almost linearly from $\approx 2.69$ minutes $(L=50 \mathrm{Mm})$ to $\approx 21.34$ minutes $(L=400 \mathrm{Mm})$, when all sinks of energy are switched on (solid line). At higher temperatures, however, the linear behavior changes slope at $L=100 \mathrm{Mm}(T=8 \mathrm{MK})$ and at $L=200 \mathrm{Mm}(T=10 \mathrm{MK})$ because of the overwhelming viscous effects in short loops, which induce similar changes of slope at such lengths (longdashed lines). Compared to the undamped waves (dotted lines), thermal conduction (short-dashed lines) increases the periods by about 30\% regardless of the loop length and temperature. The closeness between the short-dashed and the solid lines indicates that the periods of damped standing slow waves are mostly affected by thermal conduction.

The decay time of the velocity perturbation for models with $d>0$, defined by

$$
\tau_{\mathrm{d}}=\frac{P}{\ln \left(\frac{v_{0}}{v_{1}}\right)},
$$

is plotted in the right panels of Figure 1 . In these plots, the solid lines describe the dependence of the decay time on loop length when both $\varepsilon>0$ and $d>0$, while the long-dashed and short-dashed curves correspond to the cases when either only viscous or thermal conductive effects are included, respectively. In the above relation, $P$ denotes the first period of oscillation, while $v_{0}$ and $v_{1}$ are, respectively, the velocity amplitudes at the beginning and after completion of the first period. In the presence of viscous and conductive sources of dissipation (i.e., $\varepsilon>0$ and $d>0$ ), the damping time increases with the loop length and decreases with the temperature. In the $T=6.3 \mathrm{MK}$ loops, the linear analysis predicts that the decay time rises from $\approx 1.02$ minutes when $L=50 \mathrm{Mm}$ to $\approx 27.62$ minutes when $L=400 \mathrm{Mm}$. Since both $\varepsilon$ and $d$ vary as $\sim T^{2}$, wave dissipation is more rapid at higher temperatures. However, viscous dissipation (long-dashed lines) 

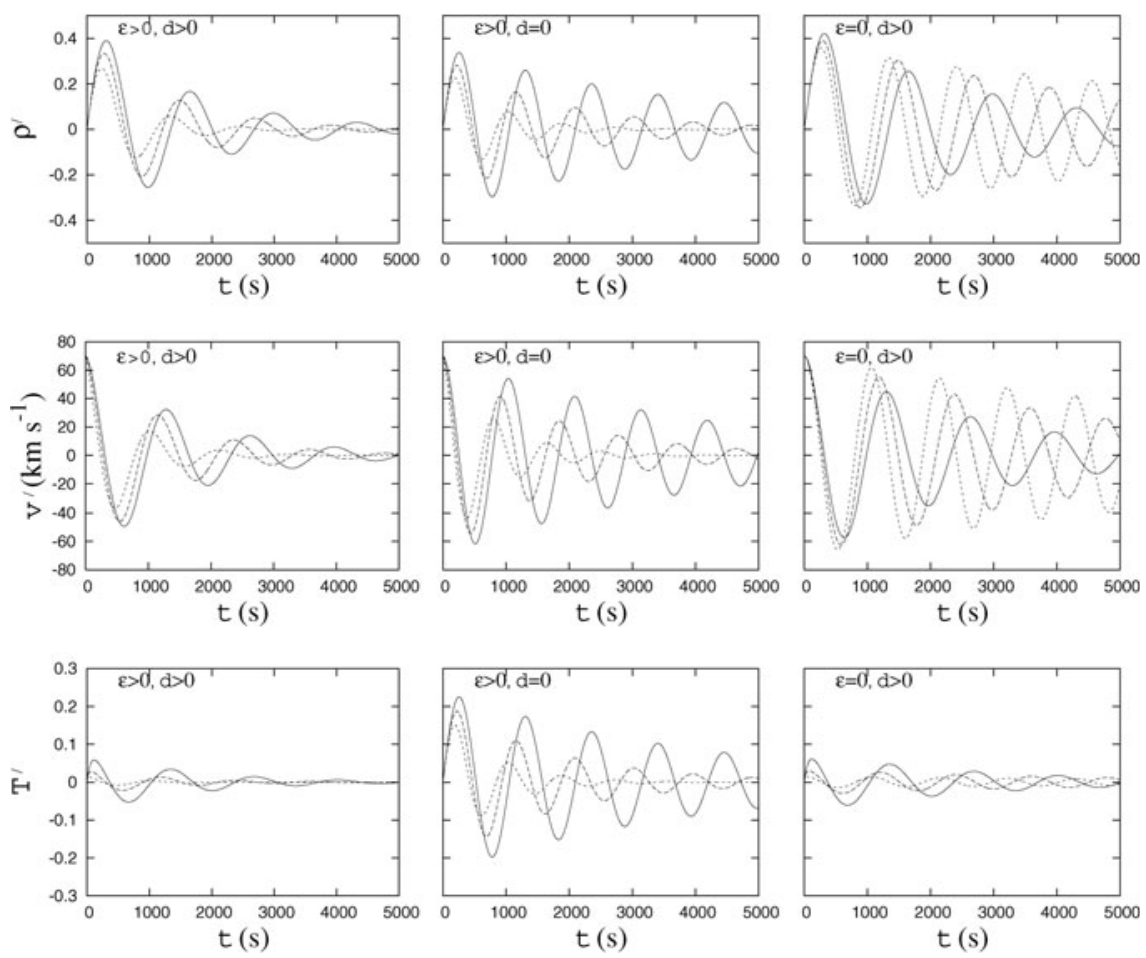

Figure 2. Temporal evolution of the density, velocity, and temperature oscillations at $\tilde{s}=0.35$ in a loop of length $L=400 \mathrm{Mm}$. The initial velocity wave amplitude is $86.6 \mathrm{~km} \mathrm{~s}^{-1}$. The solid, long-dashed, and short-dashed curves apply to loop temperatures of $6.3,8$, and $10 \mathrm{MK}$, respectively. The left panels depict the evolution when both $\varepsilon>0$ and $d>0$, while the central and right panels display the wave evolution when $d=0$ (no thermal conduction) and $\varepsilon=0$ (no viscosity), respectively.

produces a much rapid decay of the velocity wave than does thermal conduction (shortdashed lines). In particular, the decay times obtained from thermal conduction alone are between $\approx 44-49$ minutes when $T=6.3 \mathrm{MK}$ and $\approx 139-141$ minutes when $T=10$ MK. In contrast, the decay times obtained from compressive viscosity are between $\approx 1.04$ -67 minutes for $T=6.3 \mathrm{MK}$ and $\approx 0.83-13.3$ minutes for $T=10 \mathrm{MK}$. Moreover, the closeness between the solid and long-dashed curves at small lengths for $T=6.3 \mathrm{MK}$ and, in general, at all lengths for $T \geqslant 8 \mathrm{MK}$ indicates that, unlike the period, the damping time is shaped by the effects of compressive viscosity. Only at low temperatures, of the order of $6.3 \mathrm{MK}$ or less, do the concurrent effects of thermal conduction affect the damping time in long $(L>300 \mathrm{Mm})$ loops. In passing, we note that in the linear limit both the period and the decay time are independent of the size of the initial velocity perturbation amplitude $v_{0}$.

\subsubsection{Stratified Loops}

In a recent paper, Roberts (2006) demonstrated that slow wave behavior can be extracted from the MHD equations and separated from the fast magnetoacoustic wave. For the special case of a uniform and vertical magnetic field embedded in a vertically stratified medium, where $\mathbf{g}=-g_{\odot} \mathbf{z}$, he found that the slow mode is entirely described by the Klein-Gordon equation. Application of his analysis to standing slow waves in hot SUMER loops predicts a reduction of the wave period due to stratification by the amount $\left[1+\left(L / L_{\mathrm{c}}\right)^{2}\right]^{1 / 2}$ for the principal $n=1$ mode, where $L_{\mathrm{c}} \sim 2 \pi \mathcal{H}_{0}$ is an effective length 

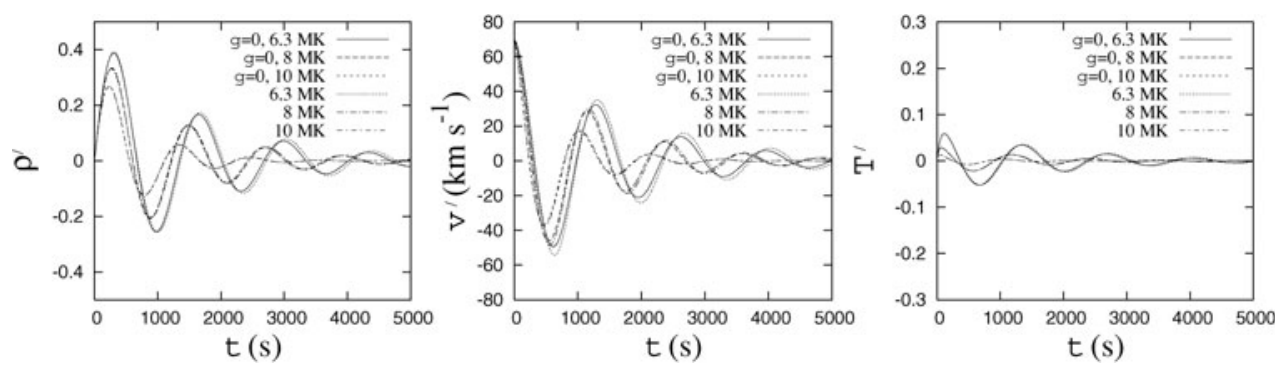

Figure 3. Temporal evolution of the density, velocity, and temperature waves at $\tilde{s}=0.35$ in a loop of length $L=400 \mathrm{Mm}$ for $\varepsilon>0, d>0$, and an initial wave-velocity amplitude of $86.6 \mathrm{~km}$ $\mathrm{s}^{-1}$. The wave evolution in a stratified loop is compared to that in a nonstratified loop for varying temperatures. The solid $(T=6.3 \mathrm{MK})$, long-dashed $(T=8 \mathrm{MK})$, and short-dashed $(T=10$ MK) curves apply to nonstratified loops, while the dotted $(T=6.3 \mathrm{MK})$, dot-long-dashed $(T=8 \mathrm{MK})$, and dot-short-dashed $(T=10 \mathrm{MK})$ curves apply to stratified loops.

introduced by stratification and $\mathcal{H}_{0}=c_{\mathrm{s}}^{2} /\left(\gamma g_{\odot}\right)$. In general, for hot coronal loops $L / L_{\mathrm{c}}$ is a small factor, implying that the effects of gravitational stratification on wave period are also small.

When only gravity is taking into account the eigenfrequencies are given by

$$
\omega_{n}=n \pi\left[1+\frac{1}{\left(2 n \pi \tilde{\mathcal{H}}_{0}\right)^{2}}\right]^{1 / 2},
$$

If we identify $2 \pi \tilde{\mathcal{H}}_{0}$ with the dimensionless effective loop length $L_{\mathrm{c}} / L$ and set $n=$ 1 , we recover the results found by Roberts (2006). Since for our hot loop parameters $5 \lesssim L_{\mathrm{c}} / L \lesssim 64$, gravitational stratification produces only a small increase of the wave frequency and therefore a small reduction of the wave period. In particular, for the second spatial harmonic $(n=2)$, the modified period is

$$
P=\tau_{\mathrm{s}}\left[1+\frac{1}{\left(4 \pi \tilde{\mathcal{H}}_{0}\right)^{2}}\right]^{-1 / 2} .
$$

For $n=2$ the dimensionless effective length is twice as large, implying that the magnitude of $\left(L / L_{\mathrm{c}}\right)^{2}$ is one-fourth smaller than for $n=1$.

When viscosity and gravity is considered together for $n=2$ the modified period due to the effects of stratification and viscous dissipation is

$$
P=\tau_{\mathrm{s}}\left\{1+\frac{1}{\left(4 \pi \tilde{\mathcal{H}}_{0}\right)^{2}}-\frac{(4 \pi \varepsilon)^{2}}{9}\left[1-\frac{1}{2\left(2 \pi \tilde{\mathcal{H}}_{0}\right)^{2}}\right]^{2}\right\}^{-1 / 2}
$$

while the decay time becomes

$$
\tau_{\mathrm{d}}=\frac{3 \tau_{\mathrm{s}}}{\varepsilon\left(8 \pi^{2}-\frac{1}{\tilde{\mathcal{H}}_{0}^{2}}\right)} .
$$

In particular, Equation (3.7) predicts periods that are $\sim 0.01 \%(L=50 \mathrm{Mm})$ to $\sim 0.5 \%$ $(L=400 \mathrm{Mm})$ smaller compared to Equation (3.3) when $T=6.3 \mathrm{MK}$. For hotter loops the reduction becomes even smaller. Conversely, stratification increases the decay times due to compressive viscosity by negligible amounts in the short loops, while for the $L=400 \mathrm{Mm}$ loops the decay times are $\sim 0.8 \%(T=10 \mathrm{MK})$ to $\sim 2 \%(T=6.3 \mathrm{MK})$ longer compared to the nonstratified case. 
The effects of thermal conduction are next analyzed. As for the homogeneous case, thermal conduction is seen to increase the period and decay time by a much larger amount compared to Equations (3.7) and (3.8). Essentially, the same trends depicted in Figure 1 are reproduced for the stratified loops. Perhaps the most significant point here is that under gravity, thermal conduction leads to an additional increase of both the period and decay time compared to the homogeneous loops. To explain the increase of the wave period let us consider the velocity wave equation when also thermal conduction is switched on

$$
\frac{\partial^{2} v^{\prime}}{\partial \tilde{t}^{2}}-\frac{\partial^{2} v^{\prime}}{\partial \tilde{s}^{2}}=-\frac{1}{\tilde{\mathcal{H}}}\left[\frac{\partial v^{\prime}}{\partial \tilde{s}}-\frac{v^{\prime}}{\gamma \tilde{\mathcal{H}}} \frac{d \tilde{\mathcal{H}}}{d \tilde{s}}\right]+\frac{4}{3} \varepsilon \frac{\partial}{\partial \tilde{t}}\left(\frac{\partial^{2} v^{\prime}}{\partial \tilde{s}^{2}}\right)+d\left(\frac{1}{\tilde{\mathcal{H}}} \frac{\partial^{2} T^{\prime}}{\partial \tilde{s}^{2}}-\frac{\partial^{3} T^{\prime}}{\partial \tilde{s}^{3}}\right)
$$

We see that the term $(d / \tilde{\mathcal{H}})\left(\partial^{2} T^{\prime} / \partial \tilde{s}^{2}\right)$ couples the gravitational field to the effects of thermal conduction. Note that it vanishes in the absence of gravity $(\tilde{\mathcal{H}} \rightarrow \infty)$. This term is in fact responsible for the additional increase of wave period due to stratification when thermal conduction is included.

The effects of stratification on wave damping are shown in Figure 3 (at $\tilde{s}=0.35$ ) for different temperatures in a $400 \mathrm{Mm}$ loop model with $v_{0}=86.6 \mathrm{~km} \mathrm{~s}^{-1}$. At low temperatures, of the order of $6.3 \mathrm{MK}$, the density and velocity perturbations exhibit slightly larger amplitudes and longer periods (dotted lines) compared to the homogeneous case (solid lines). The difference between these waves become much smaller at higher temperatures. At $T=10 \mathrm{MK}$, they become almost undistinguishable on the scale of the plot. On the other hand, the temperature wave is essentially unaffected by stratification. Compared to the homogeneous case, stratification results in an increment of the decay time of $\approx 0.4 \%$ (for $L=50 \mathrm{Mm}$ ) to $\approx 13.4 \%$ (for $L=400 \mathrm{Mm}$ ) in the cool $(T=6.3 \mathrm{MK}$ ) loops. In the hotter $(T \geqslant 8 \mathrm{MK})$ loops, however, the oscillations damp out faster because of the larger effects of thermal conduction and compressive viscosity. In particular, for $T=10 \mathrm{MK}$ the above increments range from $\approx 0.05 \%(L=50 \mathrm{Mm})$ to $\approx 2.4 \%(L=400$ $\mathrm{Mm})$. While the present analysis applies to the linear limit, we recall that nonlinear effects may modify some of the above results.

\section{Conclusions}

In this work we have found that the major sources of wave damping are the combined effect of thermal conduction and compressive viscosity.

Thermal conduction is mostly responsible for damping of the temperature wave, while compressive viscosity is the primary source of damping of the density and velocity oscillations. We also find that thermal conduction is necessary to reproduce the observed periods, while compressive viscosity determines the observed decay times. This main result contrasts with the conclusions of previous analyses that pointed to thermal conduction as the primary mechanism for wave damping based on the realization that the viscous timescale is an order of magnitude longer than both the conductive time and the observed decay times. On the other hand, the effects of gravitational stratification are negligible, in agreement with a recent linear analysis by Roberts (2006). We also find that under stratification, thermal conduction leads to an additional increase of both the period and decay time compared to the homogeneous loops because of an inherent coupling between the gravitational field and the effects of thermal conduction.

\section{Acknowledgements}

C.A.M-B. is grateful to the CDCHT of the Universidad de los Andes for financial support (C-1367-06-05-B). The authors also thank to the IAU for the grant support. 


\section{References}

Banerjee, D. Erdélyi, R., Oliver, R., O'Shea, E. 2007, Sol. Phys., 246, 3

De Moortel, I. and Hood, A.W.: 2003, Astron. Astrophys. 408, 755.

De Moortel, I. and Hood, A.W.: 2004, Astron. Astrophys. 415, 705.

De Moortel, I., Ireland, J., and Walsh, R.W.: 2000, Astron. Astrophys. 355, L23.

Kliem, B., Dammasch, I.E., Curdt, W., and Wilhelm, K.: 2002, Astrophys. J. 568, L61.

Mendoza-Briceño, C.A., Erdélyi, R., and Sigalotti, L.Di G.: 2004, Astrophys. J. 605, 493.

Nakariakov, V.M. and Ofman, L.: 2001, Astron. Astrophys. 372, L53.

Ofman, L. and Wang, T.: 2002, Astrophys. J. 580, L85.

Roberts, B.: 2006, Roy. Soc. London Trans. Series A 364, 447.

Taroyan, Y., Erdélyi, R., Doyle, J.G., and Bradshaw, S.J.: 2005, Astron. Astrophys. 438, 713.

Taroyan, Y., Erdélyi, R., Wang, T. J. \& Bradshaw, S. J. 2007 ApJ 659, L173

Wang, T.J., Solanki, S.K., Innes, D.E., and Curdt, W.: 2005, Astron. Astrophys. 435, 753.

Wang, T.J., Solanki, S.K., Innes, D.E., Curdt, W., and Marsch, E.: 2003b, Astron. Astrophys. 402, L17.

Wang, T.J., Solanki, S.K., Curdt, W., Innes, D.E., Dammasch, I.E., and Kliem, B.: 2003a, Astron. Astrophys. 406, 1105. 